\title{
Role of Inhibition in Respiratory Pattern Generation
}

\author{
Wiktor A. Janczewski, ${ }^{1}$ Alexis Tashima, ${ }^{1}$ Paul Hsu, ${ }^{1}$ Yan Cui, ${ }^{1,2}$ and Jack L. Feldman ${ }^{1}$ \\ ${ }^{1}$ Department of Neurobiology, David Geffen School of Medicine, University of California, Los Angeles, Los Angeles, California 90095-1763, and \\ ${ }^{2}$ Department of Physiology, West China School of Preclinical and Forensic Medicine, Sichuan University, Chengdu, Sichuan 610041, People's Republic of \\ China
}

Postsynaptic inhibition is a key element of neural circuits underlying behavior, with $20-50 \%$ of all mammalian (nongranule) neurons considered inhibitory. For rhythmic movements in mammals, e.g., walking, swimming, suckling, chewing, and breathing, inhibition is often hypothesized to play an essential rhythmogenic role. Here we study the role of fast synaptic inhibitory neurotransmission in the generation of breathing pattern by blocking $\mathrm{GABA}_{\mathrm{A}}$ and glycine receptors in the preBötzinger complex (preBötC), a site essential for generation of normal breathing pattern, and in the neighboring Bötzinger complex (BötC). The breathing rhythm continued following this blockade, but the lung inflation-induced Breuer-Hering inspiratory inhibitory reflex was suppressed. The antagonists were efficacious, as this blockade abolished the profound effects of the exogenously applied GABA $\mathrm{A}_{\mathrm{A}}$ receptor agonist muscimol or glycine, either of which under control conditions stopped breathing in vagus-intact or vagotomized, anesthetized, spontaneously breathing adult rats. In vagotomized rats, $\mathrm{GABA}_{\mathrm{A}}$ ergic and glycinergic antagonists had little, if any, effect on rhythm. The effect in vagus-intact rats was to slow the rhythm to a pace equivalent to that seen after suppression of the aforementioned Breuer-Hering inflation reflex. We conclude that postsynaptic inhibition within the preBötC and BötC is not essential for generation of normal respiratory rhythm in intact mammals. We suggest the primary role of inhibition is in shaping the pattern of respiratory motor output, assuring its stability, and in mediating reflex or volitional apnea, but not in the generation of rhythm per se.

\section{Introduction}

Rhythmic movements in mammals, e.g., walking, swimming, suckling, chewing, and breathing, involve alternating activation of antagonistic muscles: flexors/extensors, inspiratory/expiratory, abductors/adductors. One longstanding hypothesis is that these rhythms are generated by mutually inhibitory groups of neurons [e.g. "half-centers" in the study by Brown (1914)]. Such reciprocal inhibition provides a straightforward explanation of why one phase of rhythm is silent while the other is active. This idea was adopted for breathing in an inspiratory-expiratory "half center" model (Burns, 1963), which was subsequently refined into an "off-switch" hypothesis, where high-threshold inhibitory neurons terminated the inspiratory phase (Rubio, 1972; Bradley et al., 1975; and Feldman and Cowan, 1975; Younes et al., 1978; von Euler, 1983). Subsequently, reciprocal inhibition between early inspiratory neurons and postinspiratory (post-I) neurons was postulated as the rhythmogenic mechanism (Richter, 1982; Richter et al., 1992). These models were hampered by lack of clear

Received March 31, 2012; revised Dec. 31, 2012; accepted Jan. 7, 2013.

Author contributions: W.A.J. and J.L.F. designed research; W.A.J., A.T., P.H., and Y.C. performed research; W.A.J. and P.H. analyzed data; W.A.J. and J.L.F. wrote the paper.

This work was supported by National Institutes of Health Grants HL 70029 and HL 72211. Immunohistochemistry was done by Grace Li.

Correspondence should be addressed to Dr. Wiktor A. Janczewski, Department of Neurobiology, David Geffen School of Medicine, P.0. Box 951763, 10833 LeConte Avenue, Los Angeles, CA 90095-1763. E-mail: victoraj@mednet.ucla.edu.

A. Tashima's present address: Penn State Hershey College of Medicine, Hershey, PA 17033.

P. Hsu's present address: Department of Cellular and Molecular Physiology, Penn State Hershey College of Medicine, Hershey, PA 17033.

DOI:10.1523/JNEUROSCI.1595-12.2013

Copyright $\odot 2013$ the authors $\quad 0270-6474 / 13 / 335454-12 \$ 15.00 / 0$ identification of brainstem structures and associated neurons critical for rhythmogenesis. The identification of the Bötzinger complex (BötC) (Kalia et al., 1979) and demonstration that it contained several populations of inhibitory neurons, including postinspiratory and augmenting expiratory (Fedorko and Merrill, 1984; Ezure et al., 2003a,b; Smith et al., 2007), and of the preBötzinger complex (preBötC) (Feldman et al., 1990; Smith et al., 1991), which contains excitatory neurons critical for normal breathing in intact mammals (Tan et al., 2008) as well as a substantial population of inhibitory neurons (Winter et al., 2009), provided a basis for further refinement of models where inhibition was critical for rhythmogenesis. In a current model of this type, an inhibitory "ring" of early inspiratory neurons in the preBötC and postinspiratory neurons and augmenting expiratory neurons in the BötC (Smith et al., 2007, their Fig. $8 \mathrm{~A}$ ) is proposed to generate normal respiratory rhythm in intact mammals (Smith et al., 2007, 2012). This model predicts that blockade of inhibition at its proposed sites of action the preBötC and BötC should produce apnea (Smith et al., 2007, their Figs. 12, 13C).

To evaluate the role of fast synaptic $\left(\mathrm{GABA}_{\mathrm{A}^{-}}\right.$and glycinemediated) inhibition in generation of respiratory pattern, we injected effective antagonists into both the preBötC and BötC in anesthetized, spontaneously breathing adult rats. In vagotomized rats, breathing frequency did not change. In vagus-intact rats, frequency slowed to that of vagotomized rats, while the BreuerHering inspiratory inhibitory inflation reflex was suppressed. A complementary strategy to suppress inhibition was to ablate a critical source in the BötC, with the result that a normal breathing pattern continued. We conclude that postsynaptic inhibition within the preBötC and BötC is not essential for generation of 

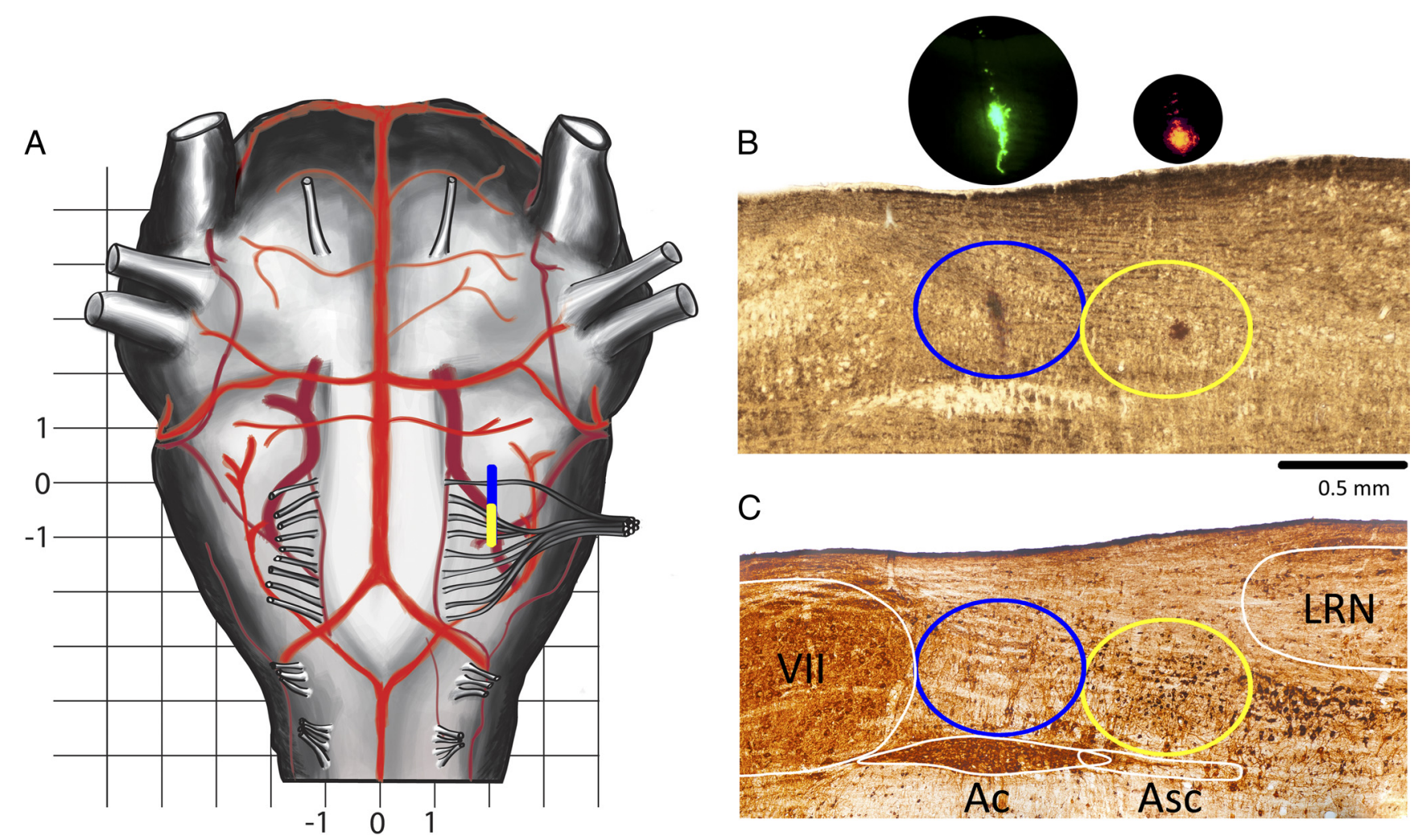

C

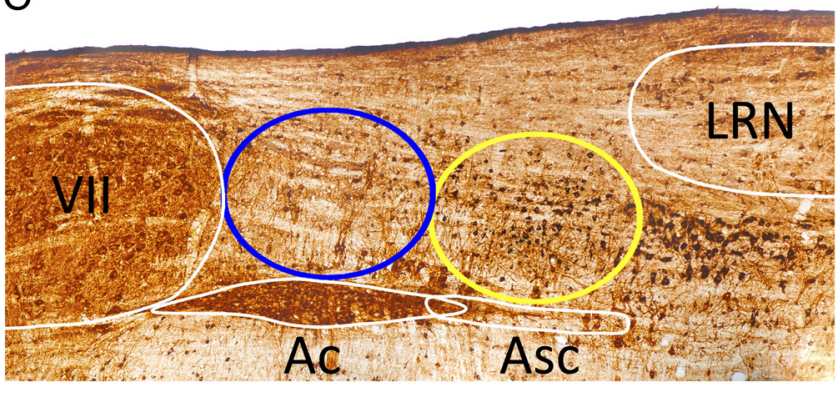

Figure 1. Histology. $A$, Schema of ventral brainstem surface. Rostrocaudal extension of the preBötC/BötC is marked by a yellow/blue bar. Grid scale, $1 \mathrm{~mm}$. Zero in the rostrocaudal direction is at the level of the most rostral root of XIIn. B, Sagittal section $2 \mathrm{~mm}$ lateral to midline; fresh wet tissue before processing. Yellow/blue ellipses encompass the preBötC/BötC. In bright field, FluoSpheres are visible as a darkened area; inserts are in dark field (DAPI filter cube). C, Nickel-enhanced reelin (brown-black) plus ChAT (light brown) double-DAB staining. Note the small ( $\sim 16 \mu \mathrm{m}$ ) neurons in the preBötC and larger $(\sim 26 \mu \mathrm{m})$ neurons just caudal to the preBötC (Tan et al., 2012). The slice in C was rescaled (1.15× rostrocaudally and $1.2 \times$ dorsoventrally) to compensate for shrinkage. Yellow/blue ellipses encompass the preBötC/BötC. VII, Facial motor nucleus.

normal respiratory rhythm in intact mammals. We suggest that the principal roles of inhibition essential for regulation of ventilation in real-world conditions in intact mammals are nonrhythmogenic and include modulation of frequency, shaping of respiratory motor output pattern and assuring its stability, control of upper airway resistance, gating of active expiration, and mediating reflex or volitional apnea.

\section{Materials and Methods}

General procedures and injections. Experimental protocols were approved by the University of California, Los Angeles, Animal Research Committee. Male Sprague Dawley rats (Charles River Farms), weighing 320-470 $\mathrm{g}$, were anesthetized with ketamine and xylazine $(100$ and $10 \mathrm{mg} / \mathrm{kg}$, respectively, i.p.). Atropine ( $0.5 \mathrm{mg} / \mathrm{kg}$, i.p.) was given to prevent bradycardia and excessive airway secretion. Isoflurane ( $1-2 \%$ vol in air) was administered throughout an experiment. The level of anesthesia was assessed by the suppression of the withdrawal reflex and by the absence of changes in heart rate and breathing rate in response to noxious stimuli. The rats were placed in a supine position in a stereotaxic instrument (David Kopf Instruments). The larynx was denervated, separated from the pharynx, and moved aside. A tracheostomy tube was placed in the trachea through the larynx; under these conditions, spontaneous breathing was unaffected by upper airway afferents. The basal aspect of the occipital bone was removed to expose the ventral aspect of the medulla.

Location of the preBötC and BötC. The preBötC and BötC have fixed positions in relation to the nucleus ambiguous subcompact (Asc) and compact (Ac), caudal pole of the facial nucleus (cVII), and lateral reticular nucleus (LRN), as established by studies using specific markers for preBötC neurons (Gray et al., 1999; Tan et al., 2012). On a parasagittal section through the preBötC as presented in Figure 1, $B$ and $C$, the rostrocaudal extent of the preBötC plus BötC (preBötC $+B o ̈ t C$ ) is $\sim 1.3 \mathrm{~mm}$, in between the cVII [corresponding to $11.85 \mathrm{~mm}$ caudal to bregma in the work of Paxinos et al. (1999, their Fig. 146)] and the LRN [corresponding to $13.1 \mathrm{~mm}$ caudal to bregma in the work of Paxinos et al. (1999, their Fig. 177)]. The BötC is ventral to $A c$, and the preBötC is ventral to Asc with the center of BötC/preBötC approximately aligned with the center of Ac/Asc (Gray et al., 1999, their Fig. 2; Smith et al., 2007, their Figs. 1, 2; Tan et al., 2010, their Fig. 9; Tan et al., 2012, their Fig. 3).

A ventral approach to the medulla is advantageous to target the preBötC $+B o ̈ t C$ due to their proximity and their position relative to easily visible protuberances of the ventral medullary surface associated with the facial nucleus and various cranial nerve roots. The most rostral root of the hypoglossal nerve (RRXII) is unique because it is thinner and separated from other roots, and thus suitable as a landmark. The entry point of the RRXII [ $12 \mathrm{~mm}$ caudal to bregma in a $274 \mathrm{~g}$ Wistar male rat in the work of Paxinos et al. (1999, their Fig. 150)] is at the same rostrocaudal level as the rostral half of the BötC. Injections targeted to the center of the BötC were made $50 \mu \mathrm{m}$ caudal to the RRXII, i.e., $300 \mu \mathrm{m}$ caudal to the $\mathrm{cVII}, 2.1 \mathrm{~mm}$ lateral to the midline, and $600 \mu \mathrm{m}$ dorsal to the ventral medullary surface. Injections targeted to the center of the preBötC were placed $750 \mu \mathrm{m}$ caudal from the RRXII, i.e., $1000 \mu \mathrm{m}$ caudal to the cVII (Fig. 1), $2 \mathrm{~mm}$ lateral to the midline, and $700 \mu \mathrm{m}$ dorsal to the ventral medullary surface. Small corrections were made to avoid puncturing of blood vessels on the surface of the medulla. Fluorescent polystyrene beads ( $2-5 \% \mathrm{vol}$ ) were added to the injectate for post hoc confirmation of injection sites. We used three types of $0.2 \mu \mathrm{m}$ FluoSpheres (Invitrogen): Orange (catalog \# F8809), Yellow-Green (catalog \#F8764), and Red (catalog \#F8763). Under fluorescent illumination with DAPI filtering, they appear yellow, green, and red, respectively.

Experimental groups. Data were obtained from 39 anesthetized adult rats $(320-470 \mathrm{~g})$ divided into seven groups. Group $1(n=5)$ and Group $2(n=5)$ tested the effectiveness of using bicuculline (B) and strychnine 
(S) in blocking synaptic inhibition in vagotomized rats (Figs. 2, 3; Table 1). Group $3(n=6)$ determined the disturbances of breathing resulting from bilateral injection of vehicle alone (110 $\mu \mathrm{l}$ of saline saline) into the preBötC and BötC. Group $4(n=7)$ tested the effects of injecting a mixture of bicuculline and strychnine $(B+S)$ into the preBötC and BötC on breathing pattern in adult anesthetized spontaneously breathing rats with intact vagi. Group $5(n=6)$ was similar to Group 4, except that rats were vagotomized (see Fig. 6; Table 2). Group $6(n=5)$ tested the effects of $\mathrm{B}+\mathrm{S}$ injections on recurrent laryngeal nerve (RLN) activity, which has a postinspiratory component. Group $7(n=5)$ tested the effect of ablation of the BötC, the region containing post-I and augmenting expiratory (aug-E) inhibitory neurons, on breathing pattern (see Fig. 8; Table 2).

Agonist-antagonist-agonist injections (Groups 1 and 2). Bicuculline (prepared from bicuculline methiodide powder; molecular weight (MW), 509.3; catalog \#2503, Tocris Bioscience] was used to antagonize $\mathrm{GABA}_{\mathrm{A}}$ receptors, and strychnine (prepared from strychnine $\mathrm{HCl}$ powder; MW, 370.9; catalog \#S8753, SigmaAldrich) was used to antagonize glycine receptors. In preliminary studies, we determined that the lowest concentration of agonist that resulted in prolonged ( $>4 \mathrm{~min}$ ) apnea in every rat was $95 \mu \mathrm{M}$ muscimol, $\sim 90 \mathrm{nl}$ per side, or 5.7 $\mathrm{mm}$ glycine $(110 \mathrm{nl})$ injected into the preBötC. The concentration of $\mathrm{B}+\mathrm{S}(238 \mu \mathrm{M}$ each) was chosen so that that there was no change in breathing when an agonist was injected after $\mathrm{B}+\mathrm{S}$. Our concentration of glycine $(5.7 \mathrm{~mm})$ was almost the same as used to produce apnea (6 mm) by Chitravanshi and Sapru (2002). Muscimol $(300 \mu \mathrm{M})$ was used to trigger apnea in the study by Bongianni et al. (2010). Various concentrations of $\mathrm{B}+\mathrm{S}$ blocked inhibition in previous studies. Pierrefiche et al. (1998) used $100 \mu \mathrm{M}$ of $\mathrm{B}+\mathrm{S}$. Bongianni et al. (2010) used 5 $\mathrm{mm}$ bicuculline and $5 \mathrm{~mm}$ strychnine. To confirm the efficiency of $\mathrm{B}+\mathrm{S}$ injections in blocking receptors, muscimol (MW, 114.1; catalog \#M1523, Sigma-Aldrich) or glycine (MW, 75.1; catalog \#S8753, Sigma-Aldrich) was injected bilaterally into the preBötC; this resulted in apnea. $\mathrm{B}+\mathrm{S}$ were then injected into the same place to restore the rhythm, and then muscimol or glycine were injected again. Agonist-antagonist-agonist injections were placed in the same spot, as verified by overlap of three different colors of FluoSpheres, one for each solution (Fig. $2 D$ ). The ventral approach facilitated multiple injections into the same spot because the entry point of a previous injection was visible in dark field under our surgical microscope.

Saline control injections (Group 3). In Group 3, instead of injecting a saline solution containing $\mathrm{B}+\mathrm{S}$, muscimol, or glycine, we injected the same volume $(110 \mu \mathrm{l})$ of saline alone. The initial injections were made bilaterally into the preBötC $(n=6)$, and 20 min later into the preBötC+BötC $(n=6)$ in vagotomized rats. Respiratory parameters were measured before and $2 \mathrm{~min}$ after preBötC injections, then before and $2 \mathrm{~min}$ after preBötC+BötC injections (Table 1).

$B+S$ injections (Groups 4-6). $\mathrm{B}+\mathrm{S}$ were injected using micropipettes ( $\sim 40 \mu \mathrm{m}$ tip), bilaterally into the preBötC and then BötC; it took 6-10 min to complete four injections. The volume of each of the four $B+S$ injections was $110 \mu \mathrm{l}$. All injections were made using a series of pressure pulses (Picospritzer; Parker-Hannifin) applied to the open end of micropipettes. Air pressure was set to make each pulse eject $\sim 5 \mathrm{nl}$, with each of the four injections totaling $110 \mu \mathrm{l}$.

The preBötC $+B o ̈ t C$ spans $\sim 1.3 \mathrm{~mm}$. After collecting data for analysis, in more than half of rats we performed two additional injections within the preBötC $+\mathrm{Böt} C$ on each side. Injections were placed "in between" the original ones to test whether there was any advantage to covering the preBötC $+\mathrm{Böt} C$ with four injections per side instead of two injections per side; there was no additional respiratory response.

Ablation of the BötC (Group 7). We ablated the BötC, a primary source of inhibition to the whole respiratory network (Fedorko and Merrill, 1984; Ezure et al., 2003a,b). Bilateral BötC ablation was done using suction through a hypodermic needle $(n=5)$. The resulting lesion was $\sim 700$ $\mu \mathrm{m}$ in diameter and centered at $50 \mu \mathrm{m}$ caudal to the level of the RRXII, i.e., $300 \mu \mathrm{m}$ caudal to the $\mathrm{cVII}$ and $2100 \mu \mathrm{m}$ lateral to the midline. All tissue ventral to Asc was removed bilaterally. When blood vessels on the surface of the medulla prevented perpendicular placement of the suction needle, we approached the BötC at an angle from a more medial position. Ablation extended $\sim 300 \mu \mathrm{m}$ rostral and $400 \mu \mathrm{m}$ caudal to the RRXII and resulted in total destruction of the BötC in every rat (Fig. 8). There was 
A

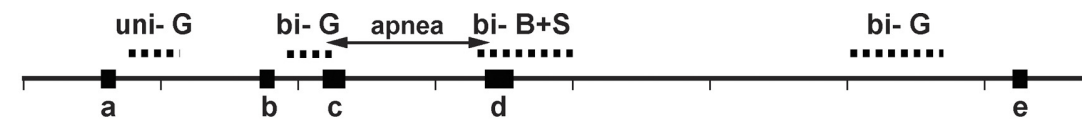

B

a) control

ธ

c) bi- $\mathrm{G}$ results in apnea b) uni- G
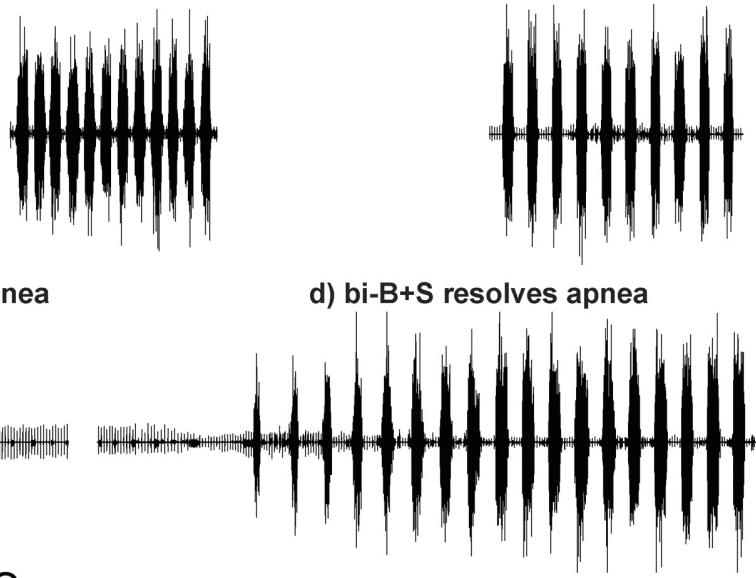

C

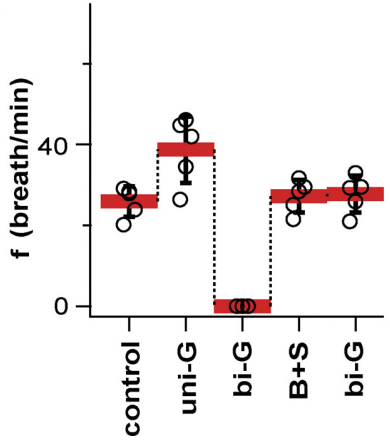

e) bi- $\mathbf{G}$ after $B+S$

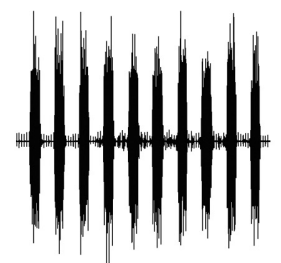

d) bi-B+S resolves apnea

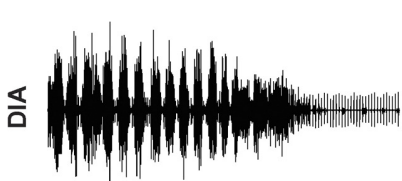


Table 1. Control injections; respiratory parameters in response to agonist-antagonist-agonist and saline injections into the preBötC

\begin{tabular}{|c|c|c|c|c|}
\hline & $f$ (breaths/min) & $V_{\mathrm{T}}(\mathrm{ml})$ & $T_{1}(s)$ & $T_{\mathrm{E}}(\mathrm{s})$ \\
\hline \multicolumn{5}{|l|}{ Group $1(n=5)$} \\
\hline \multirow{3}{*}{ a: Control } & $26.6 \pm 6.5$ & $3.9 \pm 0.5$ & $0.7 \pm 0.2$ & $1.7 \pm 0.3$ \\
\hline & $a=c=d$ & $a=c=d$ & $\mathrm{a}=\mathrm{b}=\mathrm{c}=\mathrm{d}$ & $\mathrm{a}=\mathrm{c}=\mathrm{d}$ \\
\hline & $\begin{array}{l}F_{(2,8)}=1.029 ; p=0.400 \\
51.9 \pm 18.2\end{array}$ & $\begin{array}{l}F_{(2,8)}=4.348 ; p=0.053 \\
2.3 \pm 0.7\end{array}$ & $\begin{array}{l}F_{(3,12)}=2.562 ; p=0.104 \\
0.6 \pm 0.1\end{array}$ & $\begin{array}{l}F_{(2,8)}=3.259 ; p=0.092 \\
0.7 \pm 0.4\end{array}$ \\
\hline b: Excitatory response to $\mathrm{M}$ & $\begin{array}{l}b>a=c=d \\
F_{(3,12)}=12.887 ; p=0.001\end{array}$ & $\begin{array}{l}\mathrm{b}<\mathrm{a}=\mathrm{c}=\mathrm{d} \\
\mathrm{F}_{(3,12)}=27.874 ; p=0.001\end{array}$ & & $\begin{array}{l}\mathrm{b}<\mathrm{a}=\mathrm{c}=\mathrm{d} \\
F_{(3,12)}=17.101 ; p=0.001\end{array}$ \\
\hline$c: M+(B+S)$ & $\begin{array}{l}29.8 \pm 5.2 \\
c=d(p=0.900)\end{array}$ & $\begin{array}{l}3.5 \pm 0.7 \\
c=d(p=0.982)\end{array}$ & $0.6 \pm 0.3$ & $\begin{array}{l}1.5 \pm 0.1 \\
c=d(p=0.625)\end{array}$ \\
\hline$d: M+(B+S)+M$ & $27.5 \pm 2.4$ & $3.4 \pm 0.7$ & $0.5 \pm 0.2$ & $1.7 \pm 0.3$ \\
\hline \multicolumn{5}{|l|}{ Group $2(n=5)$} \\
\hline \multirow{3}{*}{ a: Control } & $25.9 \pm 3.8$ & $3.9 \pm 0.4$ & $0.8 \pm 0.2$ & $1.6 \pm 0.3$ \\
\hline & $\mathrm{a}=\mathrm{c}=\mathrm{d}$ & $\mathrm{a}=\mathrm{c}=\mathrm{d}$ & $\mathrm{a}=\mathrm{b}$ & $\mathrm{a}=\mathrm{c}=\mathrm{d}$ \\
\hline & $\begin{array}{l}F_{(2,8)}=0.884 ; p=0.450 \\
38.8 \pm 8.2\end{array}$ & $\begin{array}{l}F_{(2,8)}=0.917 ; p=0.438 \\
2.2 \pm 0.4\end{array}$ & $\begin{array}{l}F_{(1,4)}=3.152 ; p=0.150 \\
0.7 \pm 0.2\end{array}$ & $\begin{array}{l}F_{(2,8)}=0.859 ; p=0.459 \\
0.9 \pm 0.3\end{array}$ \\
\hline b: Excitatory response to $\mathrm{G}$ & $\mathrm{b}>\mathrm{a}=\mathrm{c}=\mathrm{d}$ & $\mathrm{b}<\mathrm{a}=\mathrm{c}=\mathrm{d}$ & $\mathrm{b}=\mathrm{a}>\mathrm{c}=\mathrm{d}$ & $\mathrm{b}<\mathrm{a}=\mathrm{c}=\mathrm{d}$ \\
\hline \multirow[t]{2}{*}{$c: G+(B+S)$} & $\begin{array}{l}F_{(3,12)}=19.198 ; p=0.001 \\
27.2 \pm 4.0\end{array}$ & $\begin{array}{l}F_{(3,12)}=26.936 ; p=0.001 \\
3.6 \pm 0.4\end{array}$ & $\begin{array}{l}F_{(3,12)}=10.727 ; p=0.001 \\
0.6 \pm 0.1\end{array}$ & $\begin{array}{l}F_{(3,12)}=29.704 ; p=0.001 \\
1.7 \pm 0.4\end{array}$ \\
\hline & $c=d ; p=0.992$ & $c=d ; p=0.981$ & $c=d ; p=0.988$ & $c=d ; p=0.833$ \\
\hline$d: G+(B+S)+G$ & $27.7 \pm 4.5$ & $3.7 \pm 0.5$ & $0.6 \pm 0.1$ & $1.7 \pm 0.3$ \\
\hline \multicolumn{5}{|l|}{ Group $3(n=6)$} \\
\hline \multirow[b]{2}{*}{ a: Before saline injection into preBötC } & $30.3 \pm 4.5$ & $4.1 \pm 0.7$ & $0.5 \pm 0.2$ & $1.5 \pm 0.4$ \\
\hline & $\begin{array}{l}\mathrm{a}=\mathrm{b}=\mathrm{c}=\mathrm{d} \\
F_{(3,15)}=0.801 ; p=0.512\end{array}$ & $\begin{array}{l}\mathrm{a}=\mathrm{b}=\mathrm{c}=\mathrm{d} \\
F_{(3,15)}=1.077 ; p=0.389\end{array}$ & $\begin{array}{l}\mathrm{a}=\mathrm{b}=\mathrm{c}=\mathrm{d} \\
F_{(3,15)}=2.562 ; p=2.262\end{array}$ & $\begin{array}{l}\mathrm{a}=\mathrm{b}=\mathrm{c}=\mathrm{d} \\
F_{(3,15)}=1.300 ; p=0.311\end{array}$ \\
\hline b: 2 min after saline injection into preBötC & $30.6 \pm 5.3$ & $3.9 \pm 0.5$ & $0.5 \pm 0.1$ & $1.5 \pm 0.4$ \\
\hline c: Before saline injection into preBötC + BötC & $30.8 \pm 5.4$ & $4.0 \pm 0.5$ & $0.5 \pm 0.1$ & $1.5 \pm 0.4$ \\
\hline $\mathrm{d}: 2$ min after saline injection into preBötC + BötC & $31.7 \pm 5.0$ & $3.6 \pm 0.6$ & $0.5 \pm 0.1$ & $1.4 \pm 0.4$ \\
\hline
\end{tabular}

The $F$ statistics from repeated-measures ANOVA are reported as $F_{\left(\mathrm{df}_{\mathrm{B} T}, \mathrm{df} \mathrm{f}_{\mathrm{B}}\right)}=F$ value, where $\mathrm{df}_{\mathrm{BT}}$ is the degrees of freedom between subjects, and df $\mathrm{f}_{\mathrm{R}}$ is the degrees of freedom for residuals. ANOVA was followed by all pairwise multiple comparison procedure (Tukey test). Notation $\mathrm{a}=\mathrm{b}=\mathrm{c}$ means that when a respiratory parameter was measured in the same group of rats under conditions $\mathrm{a}$, $\mathrm{b}$, and $\mathrm{c}$, the differences in the mean values were not great enough to exclude the possibility that the difference was due to random sampling variability, i.e., means were not statistically different. Notation $\mathrm{a}>\mathrm{b}=\mathrm{c}$ means that a null hypothesis that the data under conditions $\mathrm{a}$, $\mathrm{b}$, and $\mathrm{c}$ were sampled from populations with the same mean was rejected, and subsequent pair comparison (Tukey test) established that the mean value under condition a is greater than under condition $b$, while mean values under conditions $b$ and $c$ are not significantly different. M, Muscimol; G, glycine.

Table 2. Respiratory parameters in response to vagotomy, B+S injections (Groups 4 and 5), and BötC ablation (Group 7)

\begin{tabular}{|c|c|c|c|c|}
\hline & $f$ (breath/min) & $V_{\mathrm{T}}(\mathrm{ml})$ & $T_{1}(\mathrm{~s})$ & $T_{\mathrm{E}}(\mathrm{s})$ \\
\hline \multicolumn{5}{|l|}{ Group $4(n=7)$} \\
\hline & $71.6 \pm 5.0$ & $2.0 \pm 0.5$ & $0.3 \pm 0.1$ & $0.5 \pm 0.1$ \\
\hline \multirow[t]{3}{*}{ a: Vagi intact } & $a>b>c$ & $\mathrm{a}<\mathrm{b}=\mathrm{c}$ & $\mathrm{a}<\mathrm{b}=\mathrm{c}$ & $\mathrm{a}<\mathrm{b}<\mathrm{c}$ \\
\hline & $\begin{array}{l}F_{(2,12)}=90.470 ; p=0.001 \\
a>b ; p=0.001\end{array}$ & $\begin{array}{l}F_{(2,12)}=124.428 ; p=0.001 \\
a<b ; p=0.001\end{array}$ & $\begin{array}{l}F_{(2,12)}=21.548 ; p=0.001 \\
a<b ; p=0.002\end{array}$ & $\begin{array}{l}F_{(2,12)}=44.305 ; p=0.001 \\
a<b ; p=0.001\end{array}$ \\
\hline & $\mathrm{a}>c ; p=0.001$ & $\mathrm{a}<c ; p=0.001$ & $\mathrm{a}<c ; p=0.001$ & $\mathrm{a}<c ; p=0.001$ \\
\hline \multirow[t]{2}{*}{ b: Vagi intact plus $B+S$} & $34.4 \pm 3.7$ & $3.8 \pm 0.6$ & $0.5 \pm 0.1$ & $1.3 \pm 0.2$ \\
\hline & $\mathrm{b}>c ; p=0.004$ & $\mathrm{~b}=c ; p=0.940$ & $\mathrm{~b}=c ; p=0.205$ & $\mathrm{~b}<\mathrm{c} ; p=0.032$ \\
\hline c: Vagotomized & $28.2 \pm 4.2$ & $4.1 \pm 0.3$ & $0.6 \pm 0.1$ & $1.6 \pm 0.4$ \\
\hline \multicolumn{5}{|l|}{ Group $5(n=6)$} \\
\hline & $66.0 \pm 6.1$ & $2.2 \pm 0.5$ & $0.3 \pm 0.1$ & $0.6 \pm 0.1$ \\
\hline \multirow[t]{3}{*}{ a: Vagi intact } & $a>b=c$ & $a<b=c$ & $b>a=c$ & $\mathrm{a}<\mathrm{b}=\mathrm{c}$ \\
\hline & $\begin{array}{l}F_{(2,10)}=104.057 ; p=0.001 \\
a>b ; p=0.001\end{array}$ & $\begin{array}{l}F_{(2,10)}=43.733 ; p=0.001 \\
a<b ; p=0.001\end{array}$ & $\begin{array}{l}F_{(2,10)}=43.370 ; p=0.001 \\
a<b ; p=0.001\end{array}$ & $\begin{array}{l}F_{(2,10)}=14.173 ; p=0.001 \\
a<b ; p=0.001\end{array}$ \\
\hline & $\mathrm{a}>c ; p=0.001$ & $\mathrm{a}<c ; p=0.001$ & $a=c ; p=0.070$ & $\mathrm{a}<c ; p=0.001$ \\
\hline \multirow[t]{2}{*}{ b: Vagotomized } & $29.6 \pm 4.0$ & $4.2 \pm 0.3$ & $0.7 \pm 0.1$ & $1.3 \pm 0.3$ \\
\hline & $\mathrm{b}=c ; p=0.483$ & $\mathrm{~b}=c ; p=0.095$ & $\mathrm{~b}>c ; p=0.001$ & $\mathrm{~b}=c ; p=0.096$ \\
\hline c: Vagotomized plus B $+S$ & $33.0 \pm 8.6$ & $3.7 \pm 0.7$ & $0.5 \pm 0.1$ & $1.5 \pm 0.5$ \\
\hline \multicolumn{5}{|l|}{ Group $7(n=5)$} \\
\hline & $67.8 \pm 5.1$ & $2.5 \pm 0.6$ & $0.3 \pm 0.1$ & $0.6 \pm 0.1$ \\
\hline a: Vagi intact & $\mathrm{a}=\mathrm{b} ; F_{(1,4)}=0.163 ; p=0.707$ & $\mathrm{a}=\mathrm{b} ; F_{(1,4)}=0.836 ; p=0.412$ & $\mathrm{a}=\mathrm{b} ; F_{(1,4)}=2.647 ; p=0.179$ & $\mathrm{a}=\mathrm{b} ; F_{(1,4)}=4.341 ; p=0.106$ \\
\hline b: Vagi intact plus BötC ablation & $74.0 \pm 17.4$ & $2.2 \pm 0.7$ & $0.4 \pm 0.2$ & $0.4 \pm 0.1$ \\
\hline
\end{tabular}

For details, see Table 1 legend.

Histological processing. Histological examination was used to determine injection placement and, in Group 7, whether the BötC was completely ablated. Rats were perfused and the brainstem removed and sectioned. Freshly cut wet tissue (50 $\mu$ m coronal or sagittal sections) was photographed under white light to locate the FluoSpheres (Invitrogen) in relation to landmark structures (cVII, Asc, Ac, LRN; Fig. 1). Under white light, FluoSpheres were visible as darker (less transparent) spots (Figs. $1 B, 2 D)$. Distances from cVII, Asc, and Ac were measured. In all cases, 
A
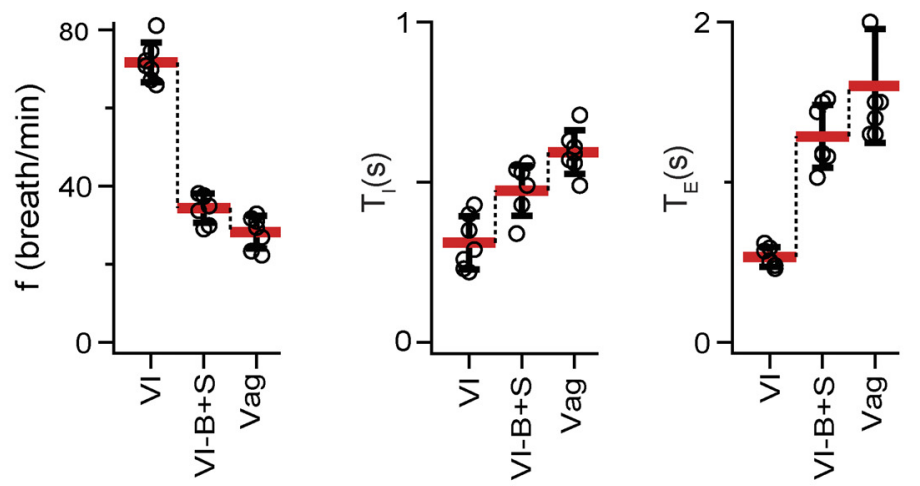

B

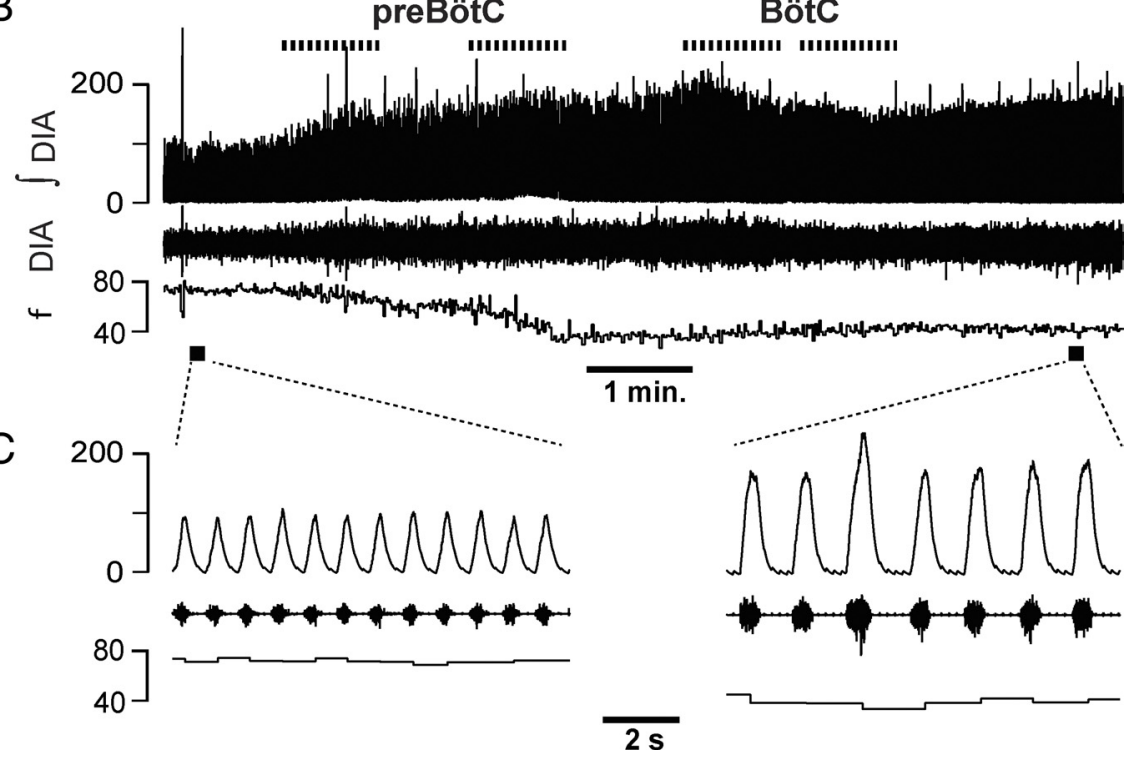

Figure 4. $B+S$ microinjection within the preBötC and BötC did not result in apnea in vagus-intact rats $(n=7) . A$, Breathing frequency $(f)$, duration of inspiration $\left(T_{1}\right)$, and duration of expiration $\left(T_{\mathrm{E}}\right)$ measured under three different conditions: control/vagi intact (VI), after administration of $B+S(\mathrm{VI}-B+S)$, and after subsequent vagotomy (Vag). $\boldsymbol{B}$, Diaphragm EMG activity and $f$ during $B+S$ injections. Top to bottom: Markers of injections; traces of integrated activity of the diaphragm ( $\left.\int D I A\right)$, raw activity of the diaphragm (DIA), and $f$; markers of expanded traces. $C$, Expanded traces in control and after $B+S$ injections.

ids 164-496 mreelin, clone G10; catalog \#MAB5364, Millipore) for $48 \mathrm{~h}$ at $4^{\circ} \mathrm{C}$, (2) secondary biotinylated donkey anti-mouse (1: 250; catalog \#715-065-150, Jackson Laboratories) antibody for $2 \mathrm{~h}$ at room temperature, (3) primary antibody anti-choline acetyltransferase (goat polyclonal; 1:500 dilution; catalog \#AB144, Millipore) overnight at room temperature, and (4) secondary antibody (biotinylated donkey anti-goat antibody; 1:400 dilution; catalog \#705-065-147, Vector Laboratories) for $2 \mathrm{~h}$ at room temperature.

\section{Results}

Control experiments: $(\mathrm{B}+\mathrm{S})$

microinjections into preBötC effectively blocked $\mathrm{GABA}_{\mathrm{A}}$ and glycine receptors (Groups 1 and 2)

We performed controls to confirm the effectiveness of blocking $\mathrm{GABA}_{\mathrm{A}}$ and glycine receptors at target sites (Figs. 2, 3). In anesthetized vagotomized rats, a micropipette filled with the $\mathrm{GABA}_{\mathrm{A}}$ agonist muscimol $(95 \mu \mathrm{M})$ was placed into the preBötC on each side $(n=5$; Group 1$)$. A sequence of pressure pulses $(\sim 5 \mathrm{nl})$ ejected $90 \mathrm{nl}$ muscimol solution per side, with bilateral injections producing apnea (during which time the rats were mechanically ventilated). After a unilateral muscimol injection, $f$ increased to $200.1 \pm$ $83.7 \%\left(F_{(1,4)}=20.985 ; p=0.010 ; n=5\right)$, and $V_{\mathbf{T}}$ dropped to $58.9 \pm 14.5 \%\left(F_{(1,4)}=\right.$ $37.674 ; p=0.004)$ of preinjection values. After bilateral injection, a similar increase in $f$ was followed by low amplitude irregular bursting, then by tonic activity and apnea (Fig. $2 B c, C$ ). We then replaced both muscimol pipettes at the same ejection sites with ones containing $\mathrm{B}+\mathrm{S}(238$ $\mu \mathrm{M}$ each). After 5-6 min of muscimolinduced apnea, we injected $\mathrm{B}+\mathrm{S}(110 \mathrm{nl}$ per side). Diaphragmatic activity and as-

these beads were found to be close $(<70 \mu \mathrm{m})$ to the center of the preBötC or BötC. They were in the same parasagittal plane as the Ac and $330 \pm 50$ $\mu \mathrm{m}$ caudal to the cVII and $260 \pm 50 \mu \mathrm{m}$ ventral to the Ac for BötC injections, and in the same parasagittal plane as the Asc and $1000 \pm 70$ $\mu \mathrm{m}$ caudal to the cVII and $260 \pm 50 \mu \mathrm{m}$ ventral to the Asc for preBötC injections.

Reelin is an effective marker for the preBötC (Tan et al., 2012); therefore, evaluation using freshly cut wet tissue was followed by double-DAB staining for reelin and choline acetyltransferase (ChAT; Fig. $1 C$ ) to confirm the accuracy of our method of finding preBötC/BötC using fresh cut sagittal slices. Slices were photographed again after a double-DAB staining. We determined that during processing, sagittal slices shrunk, on average, to $87 \%$ in the rostrocaudal direction and to $84 \%$ in the dorsoventral direction of their dimensions after cutting (Fig. 1B). For comparison, Paxinos at al. (1999) reported homogenous shrinkage to $81 \%$ of thinner $(31 \mu \mathrm{m})$ coronal slices at the level of the preBötC. After compensation for the shrinkage by a factor of 1.15 in the rostrocaudal direction and 1.2 in the dorsoventral direction (Photoshop, Adobe), images of stained and fresh slices could be overlapped to check location of the FluoSpheres in relation to reelin positive neurons marking the preBötC. We found that using the Ac, Asc, and cVII as landmarks, and by measuring distance from the cVII, the preBötC can be located with similar accuracy using fresh and processed tissue. Antibodies were used in the following sequence: (1) primary antibody (1:500; anti-reelin; amino ac- sociated breathing movements reappeared (Fig. $2 B d, C$ ), and rats were disconnected from the ventilator. Ten minutes later, bilateral injection of muscimol (identical to the one that caused apnea; $95 \mu \mathrm{M}, \sim 90 \mathrm{nl}$ per side) did not suppress breathing (Fig. $2 \mathrm{Be}$, $C$; Table 1). FluoSpheres of different colors were added during the first and second injections of muscimol and for the injection of $\mathrm{B}+\mathrm{S}$. The overlap of these distinct markers confirmed that all three injections were made within the preBötC (Fig. 2D). Group mean values of $f$ in the control, after muscimol with $B+S$, and after muscimol with $\mathrm{B}+\mathrm{S}$ and a second injection of muscimol were $26.6 \pm 6.5,29.8 \pm 5.2$, and $27.5 \pm 2.4$ breaths per minute, respectively; they did not differ $\left[F_{(2,8)}=1.029\right.$; not significant (n.s.); $p=0.400]$.

In a separate group of rats, we repeated this protocol with glycine (5.7 mM; Group 2; Fig. 3). Glycine-induced apnea was also preceded by an increase in $f$ (Fig. $3 B b, C)$. After a unilateral injection, $f$ increased to $149.4 \pm 22.9 \%\left(F_{(1,4)}=41.851 ; p=\right.$ $0.003 ; n=5)$ and $V_{\mathrm{T}}$ dropped to $57.4 \pm 6.6 \%\left(F_{(1,4)}=328.942\right.$; $p=0.001$ ) of preinjection values (Table 1 ). Bilateral injection of glycine (110 $\mathrm{nl}$ per side) induced apnea, at which point rats were ventilated. Glycine-induced apnea lasted $>4 \min (n=5)$. Apnea 
was reversed by bilateral $\mathrm{B}+\mathrm{S}$ injections (238 $\mu \mathrm{M}$ each, $110 \mathrm{nl}$ per side). A subsequent bilateral injection of the same dose of glycine that previously caused apnea $(110 \mathrm{nl} ; 5.7 \mathrm{mM})$ did not result in apnea (Fig. 3Be; Table 1). Group mean values of $f$ in the control, after glycine with $\mathrm{B}+\mathrm{S}$, and after glycine with $\mathrm{B}+\mathrm{S}$ and a second injection of glycine were $25.9 \pm 3.8$, $27.2 \pm 4.0$, and $27.7 \pm 4.5$ breaths per minute, respectively; they did not differ $\left(F_{(2,8)}=0.884\right.$; n.s.; $\left.p=0.450 ; n=5\right)$.

We also tested the effect of injecting vehicle (saline) on breathing parameters. We injected $110 \mu \mathrm{l}$ of saline first bilaterally into the preBötC alone, and $20 \mathrm{~min}$ later into the preBötC + BötC in six vagotomized rats. A single pressure pulse affected rhythm for less than five cycles. The depression of $f$ during multiple pressure pulses necessary to inject $110 \mu \mathrm{l}$ of saline varied from rat to rat. Critically, there was no significant difference in breathing just before and $2 \mathrm{~min}$ after bilateral injection of saline into the preBötC. There was no significant difference before and 2 min after completion of injections into the preBötC then BötC (Table 1; Group 3). Moreover, four series of measurements, (1) before and (2) $2 \mathrm{~min}$ after preBötC injections and (3) before and (4) $2 \mathrm{~min}$ after preBötC+BötC injections, were not statistically different (Table 1). Thus, any insult to the preBötC + BötC related to pressure pulses or to saline itself subsided within 2 min postinjection. Consequently, we collected data 2-8 min after injection.

\section{Block of inhibition in preBötC and BötC did not result in apnea but suppressed BHIR in vagus-intact rats (Group 4)}

$\mathrm{B}+\mathrm{S}$ was injected bilaterally into the preBötC then the BötC (Figs. 4-7; Table 2). These four injections were completed within 6-10 min. In rats with intact vagi, $f$ dropped to $49.7 \pm 6.3 \%\left(F_{(1,6)}=135.859\right.$; $p=0.001 ; n=7)$ of control (i.e., preinjection) value. $T_{\mathrm{I}}$ increased to $190.4 \pm$ $29.5 \%\left(F_{(1,6)}=181.104 ; p=0.001\right)$ and expiratory duration $\left(T_{\mathrm{E}}\right)$ increased to $228.3 \pm 69.2 \%\left(F_{(1,6)}=34.699 ; p=\right.$ 0.001 ) of control (Fig. $4 A$ ). Subsequent vagotomy decreased $f$ to $81.7 \pm 7.8 \%\left(F_{(1,6)}=26.510 ; p=0.002\right)$ of that after $\mathrm{B}+\mathrm{S}$ injections, did not change $T_{\mathrm{I}}\left(F_{(1,6)}=0.106 ; p=0.755\right)$, and further increased $T_{\mathrm{E}}$ to $139.2 \pm 13.7 \%$ of that after $\mathrm{B}+\mathrm{S}$ injections $\left(F_{(1,6)}=51.790 ; p=0.001\right.$; Fig. $\left.4 A\right)$.

We further investigated why block of inhibition decreased $f$ and increased $T_{\mathrm{I}}$ and $T_{\mathrm{E}}$ in rats with intact vagi. Since vagotomy also slowed $f$, we tested the effect of block of inhibition on the BHIR, a powerful lung inflation-induced, vagally mediated reflex that requires fast synaptic inhibition within the medulla (Ezure

$\mathrm{B}$
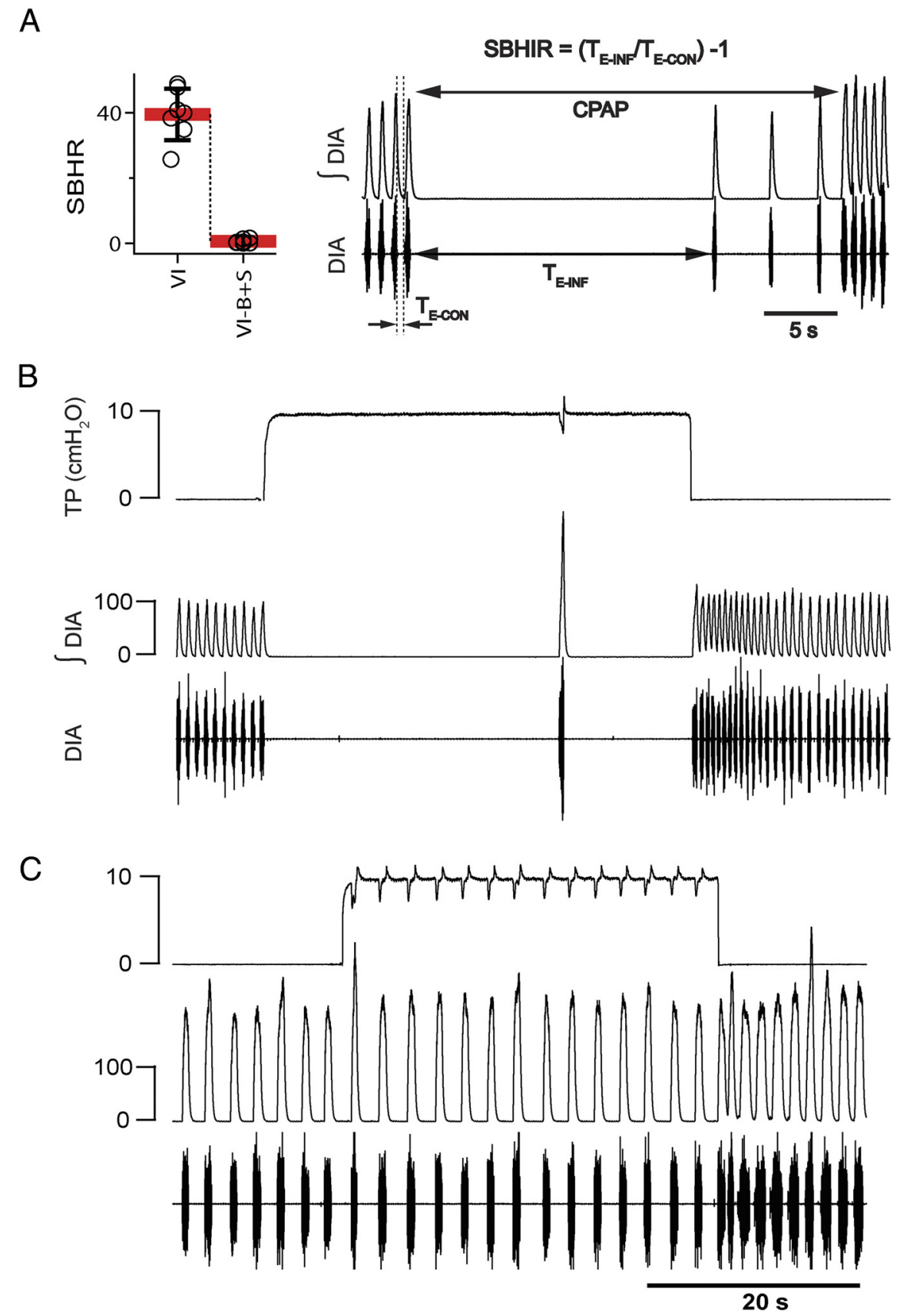

Figure 5. $B+S$ microinjection within the preBötC and BötC suppressed BHIR $(n=7) . A$, The SBHIR measured before and after $B+S$ microinjection into preBötC. The right panel explains how SBHIR was calculated. $T_{E-1 N F}$ is a period between two consecutive inspirations when lungs are inflated using $\mathrm{CPAP}\left(10 \mathrm{~cm} \mathrm{H}_{2} 0\right) ; T_{\mathrm{E}-\mathrm{CON}}$ is a period between inspirations without CPAP. SBHIR is a ratio between $T_{\mathrm{E}-\mathrm{INF}}$ and $T_{\mathrm{E}-\mathrm{CoN}}$ minus 1. $\boldsymbol{B}$, In control [vagi intact (VI)], lung inflation resulted in apnea lasting $T_{\mathrm{E}-\mathrm{INF}}=27.7 \mathrm{~s}$ and SHBIR $=42$. C, BHIR suppressed by $B+S$ within preBötC $+B o ̈ t C(V I-B+S)$. Lung inflation did not result in apnea and SHBIR $=$ $0.38,<1 \%$ of control value. Traces in $\boldsymbol{B}$ and $\boldsymbol{C}$ (top to bottom) represent tracheal pressure (TP) and integrated ( $\left.\int D I A\right)$ and raw (DIA) electromyograms of the diaphragm.

and Tanaka, 2004; Mellen et al., 2004; Kubin et al., 2006). If B+S injections suppressed the BHIR (measured by a reduction in the SBHIR; Fig. 5A), this could at least partially explain their effects on $f$. Apnea induced by lung inflation $\left(T_{\mathrm{E}-\mathrm{INF}}\right)$ ranged from 14.3 to $27.8 \mathrm{~s}(19.6 \pm 3.9 \mathrm{~s} ; n=7)$, and SBHIR from 25.7 to 48.9 $(39.5 \pm 7.9 ; n=7$; Fig. $5 A)$ before $\mathrm{B}+\mathrm{S}$. After bilateral $\mathrm{B}+\mathrm{S}$ injection, the BHIR was effectively eliminated (Fig. $5 C$ ): $T_{\mathrm{E}-\mathrm{INF}}$ was reduced to $1.8 \pm 0.9 \mathrm{~s}$ (range, 0.8 to $3.2 \mathrm{~s}$ ), which was $9.4 \pm$ $5 \%$ of its preinjection value $\left(F_{(1,6)}=206.080 ; p=0.001 ; n=7\right.$; Fig. 5, compare $B, C$ ); SBHIR was $0.6 \pm 0.6$ (range, 0.07 to 1.6 ), 
A

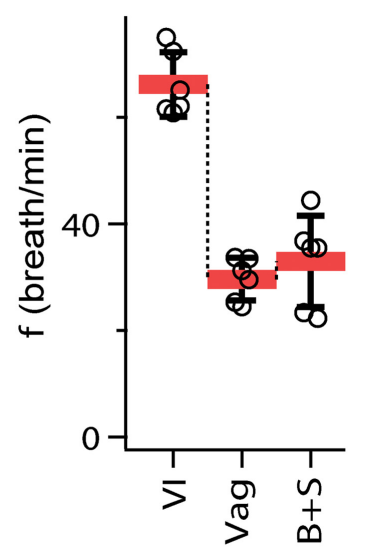

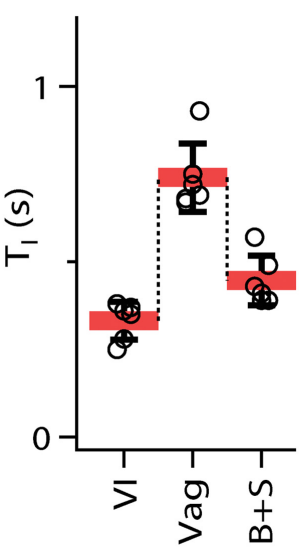

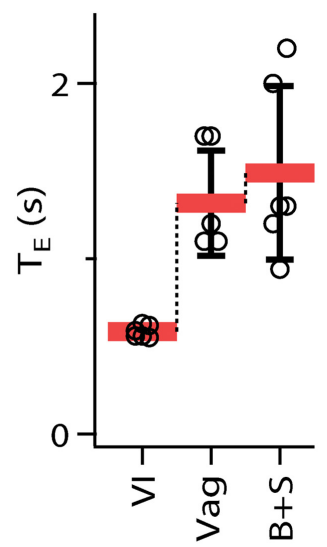

Block of inhibition in preBötC and BötC did not eliminate RLN activity in vagus-intact rats (Group 6)

In five additional rats with intact vagi, RLN activity was recorded to determine whether block of inhibition affected post-I activity (Fig. 7). The RLN had inspiratory activity followed by post-I activity during some, but not all, respiratory cycles in three of five rats before injection; in two of five rats there was no preinjection post-I activity, only tonic activity during expiration. After $\mathrm{B}+\mathrm{S}$, inspiratory RLN activity increased to $342.2 \pm 151.7 \%$ $\left(F_{(1,4)}=12.781 ; p=0.023 ; n=5\right)$, and $f$ dropped to $52.6 \pm 7.4 \%\left(F_{(1,4)}=84.259\right.$; $p=0.001 ; n=5$; Fig. 7) of control. Block of inhibition did not eliminate post-I or tonic expiratory activity $(n=5)$. In the three rats that had post-I activity during control, it increased to $730.9 \pm 231.1 \%$ $\left(F_{(1,2)}=22.379 ; p=0.042 ; n=3\right)$ of control.

Bilateral ablation of BötC did not result in apnea, nor did it abolish the BHIR in vagus-intact rats (Group 7)

The BötC contains post-I and aug-E inhibitory neurons (Ezure et al., 2003a,b). In the inhibitory ring model, these neural populations are hypothesized to be essential for normal rhythmogenesis (Smith et al., 2007; Molkov et al., 2010). Bilateral ablation of the BötC was done by exposing the ventral brainstem surface and removing the BötC by suction through a hypodermic needle $(n=5$; Fig. $8 C)$. Histological analysis confirmed ablation of the BötC plus, presumably, some surrounding tissue. Nonetheless, $f$ did not change $\left(109.9 \pm 29.9 \%\right.$ of control; $F_{(1,4)}=$ 0.163 ; n.s.; $p=0.707), T_{\mathrm{I}}$ had a tendency

Figure 6. Breathing frequency unaffected by $B+S$ microinjection within preBötC and BötC in vagotomized rats $(n=6)$. $A$, Breathing frequency $(f)$, duration of inspiration $\left(T_{1}\right)$, duration of expiration $\left(T_{\mathrm{E}}\right)$ in three different conditions: control/vagi intact $(\mathrm{VI})$, after subsequent vagotomy (Vag), and after administration of $B+S . B+S$ was injected bilaterally into preBötC then BötC $(B+S$; mean $\pm S D$, individual data points for each rat). $B$, Diaphragm EMG activity and $f$ during $B+S$ injections. From top to bottom: Markers of injections; integrated activity of the diaphragm ( $\left.\int D I A\right)$, raw activity of the diaphragm $(D I A)$, and $f$; markers of expanded traces. $C$, Expanded traces in control and after $B+S$ injections.

which was only $1.5 \pm 1.5 \%\left(F_{(1,6)}=177.410 ; p=0.001\right)$ of its value before the block. A drop of SBHIR to $<2 \%$ of its control value is comparable to the effect of blocking pulmonary stretch receptors (Davies et al., 1978). This finding links SBHIR to inhibition within preBötC $+\mathrm{BötC}$ and is a positive control for the effectiveness of the $\mathrm{B}+\mathrm{S}$ injections.

Block of inhibition in preBötC and BötC did not result in apnea in vagotomized rats (Group 5)

Vagotomy reduced $f$ to $45.2 \pm 5.4 \%\left(F_{(1,5)}=130.973 ; p=0.001 ; n=\right.$ 6) of that before vagotomy, i.e., with vagi intact (Fig. $6 \mathrm{~A}$; Table 2). After $\mathrm{B}+\mathrm{S}$ injection, $f$ did not change $(112.1 \pm 29.9 \%$; n.s.; $\left.F_{(1,5)}=0.833 ; p=0.403\right)$ compared to vagotomized controls (Fig. $6 \mathrm{~A}, C)$. $T_{\mathrm{I}}$ dropped to $61.7 \pm 29.9 \%\left(F_{(1,5)}=22.736 ; p=\right.$ 0.005). There was an insignificant trend for $T_{\mathrm{E}}$ to increase to $116.6 \pm 44.9 \%\left(F_{(1,5)}=0.645\right.$; n.s.; $\left.p=0.458\right)$, while cycle duration $\left(T_{\mathrm{I}}+T_{\mathrm{E}}\right)$ was the same $\left(95.5 \pm 30.6 \% ; F_{(1,5)}=0.833\right.$; n.s.; $p=0.403)$ as before $\mathrm{B}+\mathrm{S}$ injections. to increase $\left(142.9 \pm 38.4 \%\right.$ of control; $F_{(1,4)}=2.647 ;$ n.s.; $p=$ $0.179)$, and $T_{\mathrm{E}}$ had a tendency to decrease $(72.5 \pm 17.6 \%$ of control; $F_{(1,4)}=4.341 ;$ n.s.; $p=0.106$; Table 2, Fig. $8 A$ ). SBHIR was unaffected, equal to $43.1 \pm 2.7$ before and $48.4 \pm 8.4$ after ablations $\left(F_{(1,4)}=1.904\right.$; n.s.; $p=0.240$; Fig. $\left.8 A\right)$.

\section{Discussion}

Inhibition is critical to all aspects of nervous system function. Approximately half of the neurons in the preBötC (Winter et al., 2009) and most of the neurons in the BötC (Merrill, 1981; Fedorko and Merrill, 1984; Ezure et al., 2003a,b) are inhibitory. We hypothesized that in the intact mammal, inhibition modulates breathing frequency and can induce apnea, but is not required for rhythmogenesis underlying normal breathing, i.e., eupnea, per se. We tested this hypothesis in the adult anesthetized rat by blocking inhibition within the preBötC, which contains neurons critical for rhythmogenesis (Smith et al., 1991; Gray et al., 1999; Tan et al., 2008), and the BötC, a primary source of inhibition to the respiratory network (Merrill, 1981; Fedorko and Merrill, 1984; Feldman et al., 1984; Ezure et al., 2003a,b). We found that following block of $\mathrm{GABA}_{\mathrm{A}}$ and glycinergic receptors at 
these two sites in vagotomized rats, $f$ was unaffected (Fig. 6). In vagus-intact rats, $f$ slowed (Figs. 4, 5), which we suggest (see below) is largely a consequence of suppression of the BHIR (Fig. 5). Since B $+\mathrm{S}$ injections into preBötC + BötC did not produce apnea (nor apneusis nor gasping) and had no significant effect on $f$ in vagotomized rats, we performed two controls to assure that inhibitory neurotransmission mediated by $\mathrm{GABA}_{\mathrm{A}}$ and glycine receptors was effectively blocked. Thus, $\mathrm{B}+\mathrm{S}$ injections could (1) prevent or reverse apnea induced by preBötC injection of a GABA $_{A}$ ergic (muscimol) or a glycinergic (glycine) agonist (Figs. 2, 3), and (2) suppress the BHIR (Fig. 5). The latter effect is a positive control that also confirms a critical role of preBötC + BötC inhibition for the BHIR (Ezure and Tanaka, 2004; Mellen et al., 2004; Kubin et al., 2006) and can mostly account for the substantial reduction in $f$ following $B+S$ injections in vagus-intact rats. The increase in RLN activity in response to $\mathrm{B}+\mathrm{S}$ injections is consistent with suppression of the BHIR, because upper airway nerves (including the RLN) are strongly activated when released from the volume feedback triggering the BHIR (Kuna, 1986). Here, the post-I component of RLN activity increased approximately sevenfold, and the inspiratory component approximately threefold. An increase in post-I activity is consistent with depolarization of post-I neurons when they are released from GABAergic (Haji et al., 1992, their Fig. 6) and glycinergic inhibition (Schmid et al., 1996, their Fig. 5).

The BötC contains post-I and aug-E inhibitory neurons (Ezure et al., 2003a,b; Smith et al., 2007). Ablation of the BötC did not change $f$ in vagus-intact rats (Fig. 8 ). We conclude that the BötC is not nec-

essary for generation of respiratory rhythm; rather, its constituent post-I and aug-E inhibitory neurons modulate activity supplying upper airway muscles that control upper airway resistance and airflow (Abdala et al., 2010).

The BHIR is associated with excitation or inhibition of neurons throughout respiratory column in the medulla and pons (Hayashi et al., 1996; Kubin et al., 2006), including the BötC where post-I inhibitory neurons are excited by lung inflation (Manabe and Ezure, 1988; Fong and Potts, 2006). The persistence of the reflex after BötC lesions indicates that apnea in response to lung inflation requires inhibition within the preBötC, rather than the BötC. In en bloc preparations of neonatal rat lacking the pons and with lungs attached and innervated by the right vagus nerve, preBötC inspiratory neurons are hyperpolarized during lung inflation. Furthermore, the BHIR is eliminated by bath application of bicuculline (Mellen et al., 2004, their Figs. 4 Bii, 10C), but not by strychnine (their Fig. 7Bii), suggesting that block of $\mathrm{GABA}_{\mathrm{A}}$ (rather than glycine) receptors within the preBötC is the most likely mechanism underlying the suppression of the BHIR. Under control conditions, lung inflation could produce an apnea lasting $19.6 \pm 3.9 \mathrm{~s}(\sim 22$ respiratory cycles $)$, but following $B+S$ within the preBötC, this protective reflex was gone (Fig. 5). Since apnea is an important component of many regulatory and protective reflexes (e.g., diving and aspiration reflexes) and other motor acts (e.g., phonation, swallowing, Valsalva maneuver), we hypothesize that inhibition within the preBötC is essential for its production. We predict that $\mathrm{B}+\mathrm{S}$ injections into the preBötC will markedly alter all of these acts.

The respiratory effects of blocking inhibition in previous studies depended on experimental conditions and whether inhibition was selectively blocked in the ventral respiratory column or within the whole brainstem. In an arterially perfused in situ hypothermic brainstem-spinal cord preparation that lacks a functional BHIR, blocking of $\mathrm{Cl}^{-}$-mediated inhibition by intra-aortic perfusion with a low $\mathrm{Cl}^{-}$solution stops the rhythm (Hayashi and Lipski, 1992; Smith et al., 2007), and blocking with B+S does not stop the rhythm, which continues but changes in shape and regularity (St-John et al., 2009, their Figs. 1B, 2B). In brainstem- 
A
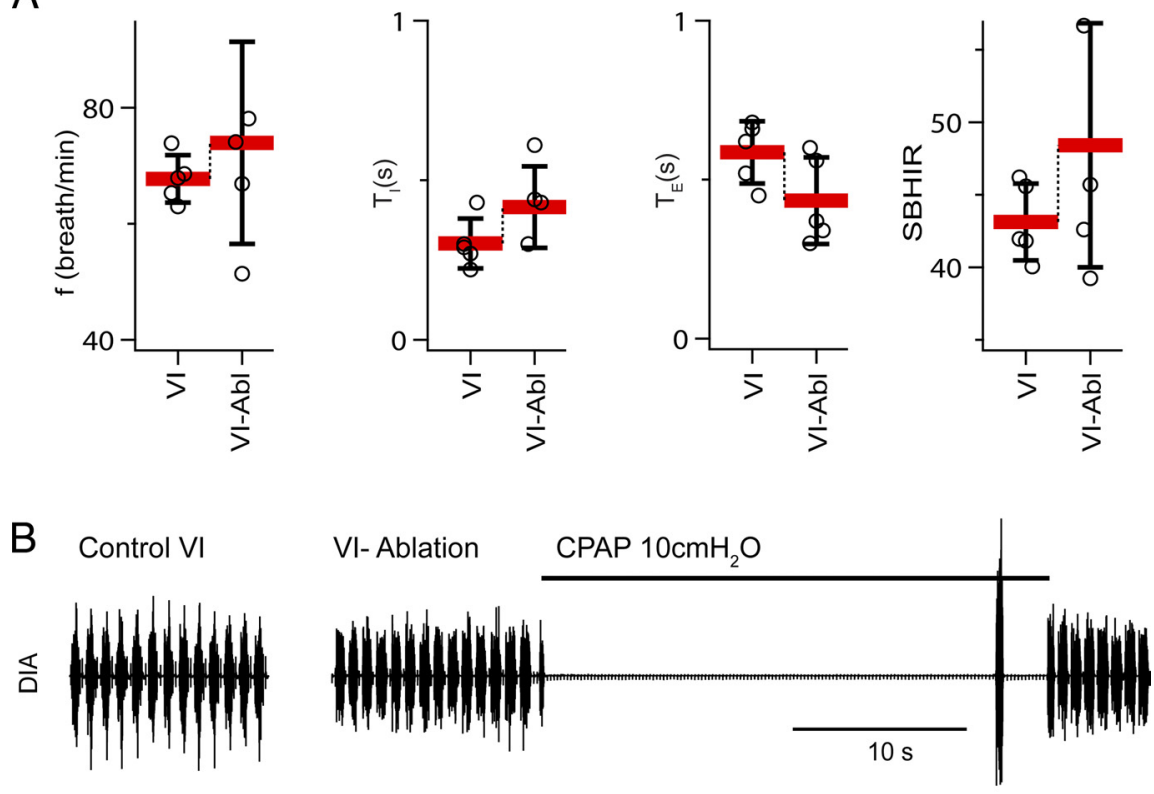

C

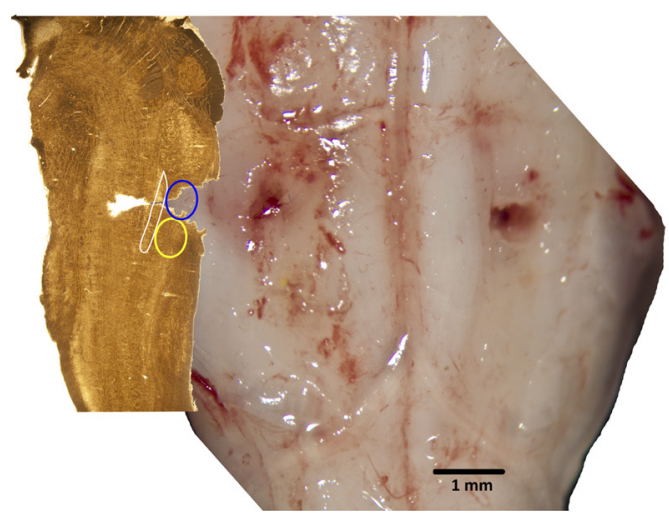

Figure 8. Ablation of the Bötzinger complex did not eliminate respiratory rhythm and did not suppress the BHIR $(n=5)$. $A$, Breathing frequency $(f)$, duration of inspiration $\left(T_{1}\right)$, duration of expiration $\left(T_{\mathrm{E}}\right)$, and $S B H I R$ before and after bilateral ablation of the BötC in rats with vagi intact $(\mathrm{VI})$. Ablation (Abl) of BötC did not diminish SBHIR (mean \pm SD; individual data points for each rat). $\boldsymbol{B}$, Activity of the diaphragm before and after bilateral BötC ablation. $C$, Histological verification of the BötC lesion. Left, Parasagittal section cut at $2.1 \mathrm{~mm}$ from the brainstem presented at the right. The blue ellipse is the presumptive location of the ablated BötC. The yellow ellipse indicates position of the preBötC.

spinal cord preparations in vitro (Feldman and Smith, 1989; Mellen et al., 2004) and medullary slices (Shao and Feldman, 1997) containing the preBötC, the rhythm continues following bath application of inhibitory antagonists. In the chloralose/ urethane-anesthetized rabbit, the rhythm continues after injection of bicuculline into the preBötC and BötC (Bongianni et al., 2010, their Figs. 6, 7). Apnea resulting from block of $\mathrm{GABA}_{\mathrm{A}}$ ergic inhibition within the BötC reverts to regular breathing following an increase in chemical drive (Bongianni et al., 2010, their Fig. 2). In pentobarbital-anesthetized adult cats, bilateral $\mathrm{B}+\mathrm{S}$ injections into the preBötC can produce apnea $(n=5$ of 7$)$, but injections into the BötC have no effect on breathing (Pierrefiche et al., 1998, their Fig. $1 A$ ). The fact that our $\mathrm{B}+\mathrm{S}$ injections into the adult rat preBötC+BötC did not produce apnea may be due to species differences or, more likely, the additional effect of systemic pentobarbital, a respiratory depressant that significantly potentiates $\mathrm{GABA}_{\mathrm{A}}$ ergic inhibition (Macdonald and Olsen, 1994) throughout the entire neuraxis, which could bias the effects of transient inhibitory blockade.

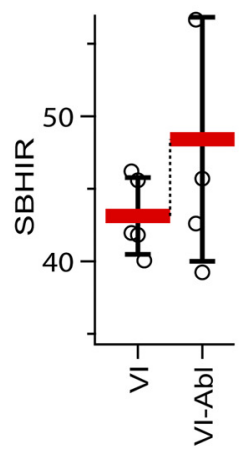

With the noted exception of pacemaker and group-pacemaker models (Feldman and Smith, 1989; Rubin et al., 2009), respiratory rhythm generation is generally postulated to critically rely on inhibition between two or three groups of neurons (Lumsden, 1923; Burns, 1963; Richter, 1982; von Euler, 1983; Richter et al., 1992; Smith et al., 2007, 2012). Results from a perfused in situ preparation led to a model where rhythm is generated by an "inhibitory ring" of three distinct neural populations that sequentially inhibit each other. In this model under normal resting conditions, excitatory preBötC neurons remain in a "tonic activity mode" and do not generate rhythm, but rather provide tonic drive that is periodically inhibited by the inhibitory ring (Smith et al., 2007; Molkov et al., 2010). When the preBötC is isolated from more rostral structures, e.g., in the rhythmic slice, the preBötC is postulated to take over generation of the rhythm by a different, pacemaker-driven mechanism ("intrinsic bursting mode") characterized by low frequency, decrementing inspiratory bursts, and absence of the post-I activity (Smith et al., 2007; St-John et al., 2009).

Could our results be explained by a rapid transformation from an inhibitiondependent rhythm to a pacemaker-driven rhythm? It is unlikely, as we did not observe a slow decrementing inspiratory motor pattern (nor gasping nor apneusis; Figs. 4-7), nor was post-I activity eliminated (Fig. 7) when inhibitory blockade was confined to sites where it could affect rhythmogenesis, i.e., the preBötC and BötC. Our data indicate that the preBötC generates rhythmic inspiratory bursts in the absence of conventional postsynaptic inhibition, with or without vagi and with or without a robust BHIR. These data can be explained by a group-pacemaker model where inspiratory bursts are generated by excitatory glutamatergic neurons endowed with activity-dependent cellular mechanisms enabling burst termination that does not require postsynaptic inhibition (Feldman and Del Negro, 2006; Krey et al., 2010; Feldman et al., 2013). We propose that inhibition, tonic or phasic, modulates burst shape (Younes et al., 1978), resulting in lower amplitude bursts and faster rhythm, as when inhibition within the preBötC was increased by muscimol (Fig. $2 B$, compare a, b) or glycine (Fig. 3B, compare a, b), and higher amplitude bursts and slower rhythm after block of inhibition (Figs. 4C, compare left, right panels; $7 B$ ).

We conclude that inhibition within the preBötC and/or BötC is not required for a normal breathing rhythm in the adult anesthetized rat, with or without vagi. We further suggest that this represents a more general principle for the role of inhibition in rhythmogenesis and central pattern generation relevant to other important movements in mammals $(\mathrm{Li}$, 2011), such as locomotion/swimming (Grillner and Jessell, 
2009; Cangiano et al., 2012) or chewing (Gossard et al., 2010). We emphasize that our conclusion does not diminish the importance of inhibition in shaping burst patterns and modulating frequency, control of airway muscles (Abdala et al., 2010), respiratory reflexes, assuring that inspiratory burst termination becomes irreversible (Younes et al., 1978), suppressing spurious excitatory sensory inputs (Feldman and Grillner, 1983), gating active expiration (Pagliardini et al., 2011), and mediating (prolonged) apneas, all essential for proper functioning of the neural circuits driving breathing under realworld conditions in intact mammals.

\section{References}

Abdala AP, Beihui HL, Rybak IA, Smith JC, Paton JF (2010) Role of GlyT2 expressing neurons in the Bötzinger complex for respiratory rhythm and pattern generation. FASEB J 24:614.3.

Bautista TG, Xing T, Fong AY, Pilowsky PM (2012) Recurrent laryngeal nerve activity exhibits a 5-HT-mediated long-term facilitation and enhanced response to hypoxia following acute intermittent hypoxia in rat. J Appl Physiol 112:1144-1156. CrossRef Medline

Bongianni F, Mutolo D, Cinelli E, Pantaleo T (2010) Respiratory responsesinduced by blockades of GABA and glycine receptors within the Bötzinger complex and the pre-Bötzinger complex of the rabbit. Brain Res 1344:134-147. CrossRef Medline

Bradley GW, von Euler C, Marttila I, Roos B (1975) A model of the central and reflex inhibition of inspiration in the cat. Biol Cybern 19:105-116. CrossRef Medline

Breuer J (1868) Self-steering of respiration through the nervus vagus in breathing (in German; E. Ullmann, translator). Reprint: Breathing: Hering-Breuer Centenary Symposium (Pother R, ed), pp 359 -394. London: J. and A. Churchill, 1970.

Brown TG (1914) On the nature of the fundamental activity of the nervous centres; together with an analysis of the conditioning of rhythmic activity in progression, and a theory of the evolution of function in the nervous system. J Physiol 48:18-46. Medline

Burns BD (1963) The central control of respiratory movements. Br Med Bull 19:7-9. Medline

Cangiano L, Hill RH, Grillner S (2012) The hemisegmental locomotor network revisited. Neuroscience 210:33-37. CrossRef Medline

Chitravanshi VC, Sapru HN (2002) Microinjections of glycine into the preBötzinger complex inhibit phrenic nerve activity in the rat. Brain Res 947:25-33. CrossRef Medline

Davies A, Dixon M, Callanan D, Huszczuk A, Widdicombe JG, Wise JC (1978) Lung reflexes in rabbits during pulmonary stretch receptor block by sulphur dioxide. Respir Physiol 34:83-101. CrossRef Medline

Ezure K, Tanaka I (2004) GABA, in some cases together with glycine, is used as the inhibitory transmitter by pump cells in the Hering-Breuer reflex pathway of the rat. Neuroscience 127:409-417. CrossRef Medline

Ezure K, Tanaka I, Kondo M (2003a) Glycine is used as a transmitter by decrementing expiratory neurons of the ventrolateral medulla in the rat. J Neurosci 23:8941-8948. Medline

Ezure K, Tanaka I, Saito Y (2003b) Brainstem and spinal projections of augmenting expiratory neurons in the rat. Neurosci Res 45:41-51. CrossRef Medline

Fedorko L, Merrill EG (1984) Axonal projections from the rostral expiratory neurones of the Bötzinger complex to medulla and spinal cord in the cat. J Physiol 350:487-496. Medline

Feldman JL, Cowan JD (1975) Large-scale activity in neural nets II: a model for the brainstem respiratory oscillator. Biol Cybern 17:39-51. CrossRef Medline

Feldman JL, Del Negro CA (2006) Looking for inspiration: new perspectives on respiratory rhythm. Nat Rev Neurosci 7:232-242. CrossRef Medline

Feldman JL, Grillner S (1983) Control of vertebrate respiration and locomotion: a brief account. Physiologist 26:310-316. Medline

Feldman JL, Smith JC (1989) Cellular mechanisms underlying modulation of breathing pattern in mammals. Ann N Y Acad Sci 563:114-130. CrossRef

Feldman JL, McCrimmon DR, Speck DF (1984) Effect of synchronous activation of medullary inspiratory bulbo-spinal neurones on phrenic nerve discharge in cat. J Physiol 347:241-254. Medline
Feldman JL, Connelly CA, Ellenberger HH, Smith JC (1990) The cardiorespiratory system within the brainstem. Eur J Neurosci [Suppl] 3:17.

Feldman JL, Del Negro CA, Gray PA (2013) Understanding the rhythm of breathing: so near, yet so far. Annu Rev Physiol 75:423-452. CrossRef Medline

Fong AY, Potts JT (2006) Neurokinin-1 receptor activation in Bötzinger complex evokes bradypnoea. J Physiol 575:869-885. CrossRef Medline

Gesell R, White F (1938) Recruitment of muscular activity and the central neurone after-discharge of hyperpnea. Am J Pysiol 122:48-56.

Gossard JP, Dubuc R, Kolta A, eds (2010) Breathe, walk and chew: the neural challenge: part I. Amsterdam: Elsevier.

Gray PA, Rekling JC, Bocchiaro CM, Feldman JL (1999) Modulation of respiratory frequency by peptidergic input to rhythmogenic neurons in the preBötzinger complex. Science 286:1566-1568. CrossRef Medline

Grillner S, Jessell TM (2009) Measured motion: searching for simplicity in spinal locomotor networks. Curr Opin Neurobiol 19:572-586. CrossRef Medline

Haji A, Takeda R, Remmers JE (1992) Evidence that glycine and GABA mediate postsynaptic inhibition of bulbar respiratory neurons in the cat. J Appl Physiol 73:2333-2342. Medline

Hayashi F, Lipski J (1992) The role of inhibitory amino acids in control of respiratory motor output in an arterially perfused rat. Respir Physiol 89:47-63. CrossRef Medline

Hayashi F, Coles SK, McCrimmon DR (1996) Respiratory neurons mediating the Breuer-Hering reflex prolongation of expiration in rat. J Neurosci 16:6526-6536. Medline

Kalia M, Feldman JL, Cohen MI (1979) Afferent projections to the inspiratory neuronal region of the ventrolateral nucleus of the tractus solitarius in the cat. Brain Res 171:135-141. CrossRef Medline

Krey RA, Goodreau AM, Arnold TB, Del Negro CA (2010) Outward currents contributing to inspiratory burst termination in preBötzinger Complex neurons of neonatal mice studied in vitro. Front Neural Circuits 4:124. Medline

Kubin L, Alheid GF, Zuperku EJ, McCrimmon DR (2006) Central pathways of pulmonary and lower airway vagal afferents. J Appl Physiol 101:618627. CrossRef Medline

Kuna ST (1986) Inhibition of inspiratory upper airway motoneuron activity by phasic volume feedback. J Appl Physiol 60:1373-1379. Medline

Li WC (2011) Generation of locomotion rhythms without inhibition in vertebrates: the search for pacemaker neurons. Integr Comp Biol 51:879889. CrossRef Medline

Lumsden T (1923) Observation on the respiratory centres in the cat. J Physiol 57:153-160. Medline

Macdonald RL, Olsen RW (1994) $\mathrm{GABA}_{\mathrm{A}}$ receptor channels. Annu Rev Neurosci 17:569-602. CrossRef Medline

Manabe M, Ezure K (1988) Decrementing expiratory neurons of the Bötzinger complex. I. Response to lung inflation and axonal projection. Exp Brain Res 72:150-158. CrossRef Medline

Mellen NM, Roham M, Feldman JL (2004) Afferent modulation of neonatal rat respiratory rhythm in vitro: cellular and synaptic mechanisms. J Physiol 556:859-874. CrossRef Medline

Merrill EG (1981) Where are the real respiratory neurons? Fed Proc 40: 2389-2394. Medline

Molkov YI, Abdala AP, Bacak BJ, Smith JC, Paton JF, Rybak IA (2010) Lateexpiratory activity: emergence and interactions with the respiratory CPG. J Neurophysiol 104:2713-2729. CrossRef Medline

Pagliardini S, Janczewski WA, Tan W, Dickson CT, Deisseroth K, Feldman JL (2011) Active expiration induced by excitation of ventral medulla in adult anesthetized rats. J Neurosci 31:2895-2905. CrossRef Medline

Paxinos G, Carrive P, Wang H, Wang P, eds (1999) Chemoarchitectonic atlas of the rat brainstem. San Diego: Academic.

Pierrefiche O, Schwarzacher SW, Bischoff AM, Richter DW (1998) Blockade of synaptic inhibition within the pre-Bötzinger complex in the cat suppresses respiratory rhythm generation in vivo. J Physiol 509:245-254. CrossRef Medline

Richter DW (1982) Generation and maintenance of the respiratory rhythm. J Exp Biol 100:93-107. Medline

Richter DW, Ballanyi K, Schwarzacher S (1992) Mechanisms of respiratory rhythm generation. Curr Opin Neurobiol 2:788-793. CrossRef Medline

Rubin JE, Hayes JA, Mendenhall JL, Del Negro CA (2009) Calciumactivated nonspecific cation current and synaptic depression promote network-dependent burst oscillations. Proc Natl Acad Sci U S A 106: 2939-2944. CrossRef Medline 
Rubio JE (1972) A new mathematical model of the respiratory center. Bull Math Biophys 34:467-481. CrossRef Medline

Schmid K, Foutz AS, Denavit-SaubiéM (1996) Inhibitions mediated by glycine and GABAA receptors shape the discharge pattern of bulbar respiratory neurons. Brain Res 710:150-160. CrossRef Medline

Shao XM, Feldman JL (1997) Respiratory rhythm generation and synaptic inhibition of expiratory neurons in pre-Bötzinger complex: differential roles of glycinergic and GABAergic neural transmission. J Neurophysiol 77:1853-1860. Medline

Smith JC, Ellenberger HH, Ballanyi K, Richter DW, Feldman JL (1991) PreBötzinger complex: a brainstem region that may generate respiratory rhythm in mammals. Science 254:726-729. CrossRef Medline

Smith JC, Abdala AP, Koizumi H, Rybak IA, Paton JF (2007) Spatial and functional architecture of the mammalian brain stem respiratory network: a hierarchy of three oscillatory mechanisms. J Neurophysiol 98:3370-3387. CrossRef Medline

Smith JC, Abdala AP, Borgmann A, Rybak IA, Paton JF (2012) Brainstem respiratory networks: building blocks and microcircuits. Trends Neurosci. Advance online publication. Retrieved January 30, 2013. doi: 10.1016/j.tins.2012.11.004. CrossRef Medline

St-John WM, Stornetta RL, Guyenet PG, Paton JF (2009) Location and properties of respiratory neurones with putative intrinsic bursting properties in the rat in situ. J Physiol 587:3175-3188. CrossRef Medline

Tan W, Janczewski WA, Yang P, Shao XM, Callaway EM, Feldman JL (2008) Silencing preBötzinger complex somatostatin-expressing neurons induces persistent apnea in awake rat. Nat Neurosci 11:538-540. CrossRef Medline

Tan W, Pagliardini S, Yang P, Janczewski WA, Feldman JL (2010) Projections of preBötzinger complex neurons in adult rats. J Comp Neurol 518:1862-1878. CrossRef Medline

Tan W, Sherman D, Turesson J, Shao XM, Janczewski WA, Feldman JL (2012) Reelin demarcates a subset of pre-Bötzinger complex neurons in adult rat. J Comp Neurol 520:606-619. CrossRef Medline

von Euler C (1983) On the central pattern generator for the basic breathing rhythmicity. J Appl Physiol 55:1647-1659. Medline

Winter SM, Fresemann J, Schnell C, Oku Y, Hirrlinger J, Hülsmann S (2009) Glycinergic interneurons are functionally integrated into the inspiratory network of mouse medullary slices. Pflugers Arch 458:459-469. CrossRef Medline

Younes MK, Remmers JE, Baker J (1978) Characteristics of inspiratory inhibition by phasic volume feedback in cats. J Appl Physiol 45: $80-86$. Medline 